\title{
REGULAR P.I.-RINGS
}

\author{
E. P. ARMENDARIZ AND JOE W. FISHER
}

\begin{abstract}
For a ring $R$ which satisfies a polynomial identity we show that the following are equivalent: (1) $R$ is von Neumann regular, (2) each two-sided ideal of $R$ is idempotent, and (3) each simple left (right) $R$-module is injective. We show that a P.I.-ring $R$ is left perfect if and only if all left $R$-modules have maximal submodules and $R$ has no infinite sets of orthogonal idempotents.
\end{abstract}

Commutative von Neumann regular rings have been characterized in various ways. However, very few of these characterizations extend to noncommutative rings. The results in this paper arose from attempting to extend to noncommutative rings a well-known theorem of Kaplansky which states that commutative regular rings are characterized by the property that all simple modules are injective. While this characterization is not valid for arbitrary rings, we prove that it does hold for a large class of rings-those which satisfy a polynomial identity (P.I.-rings). This turns out to be a corollary of a more striking result of ours which states that a P.I.-ring is von Neumann regular if and only if every two-sided ideal is idempotent.

These results are exploited in two ways. One is to show that a conjecture of Bass is valid for P.I.-rings. That is, a P.I.-ring $R$ is left perfect if and only if all left $R$-modules have maximal submodules and $R$ has no infinite sets of orthogonal idempotents. The other is to characterize semisimple Artinian P.I.-rings as those for which each completely reducible left (right) $R$-module is injective.

Throughout this paper, $R$ will denote an associative ring which does have a unity. We say that $R$ is a P.I.-ring if $R$ satisfies a polynomial identity with coefficients in the centroid and at least one coefficient is invertible.

Theorem 1. Let $R$ be a P.I.-ring. Then $R$ is von Neumann regular if and only if $I^{2}=I$ for each two-sided ideal $I$ of $R$.

Presented to the Society, January 25, 1973; received by the editors August 24, 1972 and, in revised form, September 29, 1972.

AMS (MOS) subject classifications (1970). Primary 16A30, 16A38, 16A48; Secondary 16A12, 16A52.

Key words and phrases. von Neumann regular, $V$-rings, polynomial identity, simple modules injective, completely reducible modules injective, idempotent rings, modules containing maximal submodules, perfect ring.

(c) American Mathematical Society 1973 
Proof. We will first show that for each $a \in R$ there exists a positive integer $n$ such that $R a^{n}=R a^{n+1}$. Assume to the contrary that for $a \in R$ the descending chain $\left\{R a^{i}: i=1,2, \cdots\right\}$ of left ideals does not terminate. By Zorn's lemma there exists an ideal $I$ of $R$ which is maximal with respect to the property that the chain $\left\{R a^{i}+I: i=1,2, \cdots\right\}$ does not terminate. By passing to $R / I$ we can assume that $I=0$. From Amitsur [1, Theorem 9] we obtain a positive integer $k$ for which $R a^{k}+l\left(a^{k}\right)$ contains a nonzero ideal $J$ of $R$. Then $J=J^{2} \subseteq J\left[R a^{k}+l\left(a^{k}\right)\right] \subseteq J$ implies $J=J a^{k}+J l\left(a^{k}\right)$ implies $J a^{k}=J a^{2 k}=J a^{3 k}=\cdots$. Now the chain $\left\{R a^{i}+J: i=1,2, \cdots\right\}$ terminates, i.e., there exists a positive integer $t$ such that $\left(a^{t}-x a^{t+1}\right) \in J$ for some $x \in R$. We choose a positive integer $s$ such that $s k>t+k+1$. Then $\left(a^{t}-x a^{t+1}\right) \in J$ implies that $\left(a^{t+k}-x a^{t+k+1}\right) \in J a^{k}=J a^{s k}$. Hence $a^{t+k}=$ $u a^{t+k+1}$ for some $u \in R$. By passing back to $R$ we have that the chain $\left\{R a^{i}+I: i=1,2, \cdots\right\}$ terminates and contradicts the choice of $I$. So we have established that for each $a \in R$ there exists a positive integer $n$ such that $R a^{n}=R a^{n+1}$, i.e., $R$ is left $\pi$-regular. Since $R$ is a semiprime P.I.-ring it follows from Levitzki [13, Theorem 1] that the indices of the nilpotent elements in $R$ are bounded. Wherefore $R$ is $\pi$-regular by Azumaya [3, Theorem 5]. Moreover, each prime ideal $P$ of $R$ is primitive because Posner's theorem [15] yields that $R / P$ has a primitive Artinian classical quotient ring which is forced to coincide with $R / P$ by the $\pi$-regularity of $R$. Thus $R$ and all its homomorphic images are semisimple. Therefore $R$ is regular by Kaplansky [11, Theorem 4.4]. Since the proof of the converse is clear, the proof of the Theorem is complete.

REMARK. (1) We note that with minor modifications the proof goes through for P.I.-rings without unity elements. Also it is enough to assume $I^{2}=I$ for each essential two-sided ideal $I$ of $R$. For if $J$ is any ideal in $R$, then by choosing an ideal $K$ maximal with respect to $J \cap K=0$, we obtain $J \oplus K$ essential in $R$. Hence $J \oplus K=(J \oplus K)^{2}=J^{2} \oplus K^{2}$ from which it follows that $J^{2}=J$.

(2) It follows immediately from the theorem that a P.I.-ring is von Neumann regular if it is biregular.

In Armendariz-Fisher [2] we conjectured that a necessary and sufficient condition for a P.I.-ring $R$ to be von Neumann regular is that each simple left $R$-module is injective and we gave a proof of the necessity. The following corollary provides a complete proof of that conjecture. A module is semisimple if the intersection of all its maximal submodules is zero.

COROLlaRY. Let $R$ be a P.I.-ring. Then the following are equivalent:

(a) $R$ is von Neumann regular.

(b) Each simple left (right) $R$-module is injective.

(c) Each left (right) $R$-module is semisimple. 
Proof. If (b) holds, then $L^{2}=L$ for each left ideal $L$ of $R$ by MichlerVillamayor [14, Corollary 2.2]. Therefore (a) follows from Theorem 1. That (a) implies (b) is Armendariz-Fisher [2, Theorem 3]. The equivalence of (b) and (c) is Michler-Villamayor [14, Theorem 2.1].

REMARK. Perhaps the most surprising aspect of Theorem 1 and its Corollary is the subtle strength demonstrated by the polynomial identity in forcing each left (right) ideal to be an intersection of maximal left (right) ideals on the meager assumption that two-sided ideals are idempotent.

As a consequence of an investigation into the structure of $\pi$-regular P.I.-rings, Fisher and Snider show in [7] that a semiprime P.I.-ring in which each prime ideal is maximal is a $\pi$-regular ring. We will need to make use of this fact in the next theorem which extends to P.I.-rings a commutative result discovered independently by Hamsher [9], Koifman [12], and Renault [16]. We denote the Jacobson radical of $R$ by $J(R)$.

THEOREM 2. Let $R$ be a P.I.-ring.

(a) If $R / J(R)$ is von Neumann regular and $J(R)$ is left T-nilpotent, then each left $R$-module has a maximal submodule.

(b) If each left $R$-module has a maximal submodule, then $R / J(R)$ is $\pi$ regular and $J(R)$ is left $T$-nilpotent.

Proof. As in Bass [4], $J(R)$ left $T$-nilpotent implies that $J(R) M \neq M$ for each left $R$-module $M$. Hence $\bar{M}=M / J(R) M$ is a nonzero $\bar{R}=R / J(R)$ module. Since $\bar{R}$ is regular it follows from Armendariz-Fisher [2, Theorem 3] that each simple left $\bar{R}$-module is injective. If $0 \neq x \in \bar{M}$, then there is a nonzero $\bar{R}$-homomorphism from $\bar{R} x$ onto a simple $\bar{R}$-module $S$ which by injectivity can be extended to an $\bar{R}$-homomorphism of $\bar{M}$ onto $S$. The kernel of this homomorphism is a maximal $\bar{R}$-submodule of $\bar{M}$ and hence provides a maximal $R$-submodule of $M$.

Now for the proof of (b). It follows from Bass [4, p. 470] that $J(R)$ is left $T$-nilpotent. We will show that $R / J(R)$ is $\pi$-regular by showing that each prime ideal of $R / J(R)$ is maximal and appealing to Fisher and Snider's result mentioned above. Since the property of a ring having each module containing a maximal submodule is preserved under homomorphic images of the ring, we may assume that $J(R)=0$. If $P$ is a prime ideal of $R$, then by Posner's theorem [15], $K=R / P$ has a simple Artinian classical quotient ring $Q$. We will show that $K=Q$. If not, then $Q / K$ maps onto a simple $K$-module $S$ and $S$ is $K$-isomorphic to $K / L$ for some some maximal left ideal $L$ of $K$. If $L$ is not essential in $K$, then $K$ is primitive and $K=Q$ by Kaplansky's theorem [10]. If $L$ is essential in $K$, then $L$ contains a nonzero divisor $u$ by Goldie [8, Theorem 3.9] and in turn $K u$ contains a nonzero ideal $I$ by Amitsur [1, Theorem 9]. Since $I$ is also an essential left ideal of 
$K$, it contains a nonzero divisor $v$. Then $K / L=v(K / L)=0$ which is a contradiction. Therefore $K=Q$ and every prime ideal of $R$ is maximal. Wherefore $R$ is $\pi$-regular as desired.

We can use this theorem to prove the conjecture of Bass [4, Remark (ii); p. 470] for P.I.-rings. As is well known the conjecture fails in general ([6], [12]), although it has been proven for commutative rings ([9], [12]).

TheORem 3. Let $R$ be a P.I.-ring. Then $R$ is left perfect if and only if each left $R$-module has a maximal submodule and $R$ has no infinite sets of orthogonal idempotents.

Proof. This follows immediately from Theorem 2 since $J(R)$ a nil ideal implies that finite sets of orthogonal idempotents can be lifted from $R / J(R)$ to $R$.

The examples of Cozzens [6] and Koifman [12] mentioned above have the property that all completely reducible modules are injective, yet they are not semisimple Artinian rings. However, for commutative rings injectivity of all completely reducible modules is equivalent to the ring being semisimple Artinian as has been shown by Cateforis and Sandomierski [5]. We extend this result to P.I.-rings.

TheOREM 4. Let $R$ be a P.I.-ring. Then $R$ is semisimple Artinian if and only if each completely reducible left (right) $R$-module is injective.

Proof. One way is clear. For the other way assume that all completely reducible left (right) $R$-modules are injective. By the Corollary, $R$ is regular. It will suffice to show that $R$ contains no infinite sets of orthogonal idempotents. Accordingly, let $\left\{e_{i}: i=1,2, \cdots\right\}$ be a countably infinite set of orthogonal idempotents. We have $L=R e_{1} \oplus R e_{2} \oplus R e_{3} \oplus \cdots$ and by the Corollary we can choose a maximal submodule $M_{i}$ of $R e_{i}$ for each $i$. Then $W=\oplus \sum_{i \geqq 1} R e_{i} / M_{i}$ is completely reducible and hence injective. Let $f$ be the canonical $R$-homomorphism of $L$ onto $W$. Since $W$ is injective $f$ can be extended to an $R$-homomorphism $\lambda: R \rightarrow W$. Then $\lambda(r)=r \lambda(1)$ for each $r \in R$. Clearly $\lambda(1) \in \sum_{i=1}^{k} R e_{i} / M_{i}$ for some $k$. Hence $f(L) \subseteq \sum_{i=1}^{k} R e_{i} \mid M_{i}$ which is a contradiction.

\section{REFERENCES}

1. S. A. Amitsur, Prime rings having polynomial identities with arbitrary coefficients, Proc. London Math. Soc. (3) 17 (1967), 470-486. MR 36 \#209.

2. E. P. Armendariz and Joe W. Fisher, On reduced P.I.-rings (unpublished).

3. G. Azumaya, Strongly $\pi$-regular rings, J. Fac. Sci. Hokkaido Univ. Ser. I. 13 (1954), 34-39. MR 16, 788.

4. $\mathrm{H}$. Bass, Finitistic dimension and a homological generalization of semi-primary rings, Trans. Amer. Math. Soc. 95 (1960), 466-488. MR 28 \#1212. 
5. V. C. Cateforis and F. L. Sandomierski, On commutative rings over which the singular submodule is a direct summand for every module, Pacific J. Math. 31 (1969), 289-292. MR 40 \#2665.

6. J. H. Cozzens, Homological properties of the ring of differential polynomials, Bull. Amer. Math. Soc. 76 (1970), 75-79. MR 41 \#3531.

7. J. W. Fisher and R. L. Snider, On the Von Neumann regularity of rings with regular prime factor rings (to appear).

8. A. W. Goldie, Semi-prime rings with maximum condition, Proc. London Math. Soc. (3) 10 (1960), 201-220. MR 22 \#2627.

9. R. M. Hamsher, Commutative noetherian rings over which every module has a maximal submodule, Proc. Amer. Math. Soc. 17 (1966), 1471-1472. MR 34 \#202.

10. I. Kaplansky, Rings with a polynomial identity, Bull. Amer. Math. Soc. 54 (1948), 575-580. MR 10, 7.

11. - Topological representation of algebras. II, Trans. Amer. Math. Soc. 68 (1950), 62-75. MR 11, 317.

12. L. A. Koüman, Rings over which each module has a maximal submodule, Mat. Zametki 7 (1970), 359-367= Math. Notes 7 (1970), 215-219. MR 41 \#6913.

13. J. Levitzki, A theorem on polynomial identities, Proc. Amer. Math. Soc. 1 (1950), 334-341. MR 12, 6.

14. G. Michler and $O$. Villamayor, On rings whose simple modules are injective, $\mathrm{J}$. Algebra (to appear).

15. E. C. Posner, Prime rings satisfying a polynomial identity, Proc. Amer. Math. Soc. 11 (1960), 180-183. MR 22 \#2626.

16. G. Renault, Sur les anneaux A, tels que tout A-module à gauche non nul contient un sous-module maximal, C. R. Acad. Sci. Paris Sér. A-B 264 (1967), A622-A624. MR 35 \#5472.

Department of Mathematics, University of TeXas, Austin, TeXas 78712 